\title{
Environmental pollutants modulate transcriptional activity of nuclear receptors of whales in vitro
}

Katharina Lühmann ${ }^{1,2^{*}}$, Roger Lille-Langøy ${ }^{3}$, Lene $_{\text {Oygarden }}{ }^{3}$, Kit M. Kovacs ${ }^{1}$, Christian Lydersen ${ }^{1}$, Anders Goksøyr ${ }^{3}$ and Heli Routti ${ }^{1}$ *

${ }^{1}$ Norwegian Polar Institute, Tromsø, Norway

${ }^{2}$ University Koblenz-Landau, Landau, Germany

${ }^{3}$ University of Bergen, Department of Biological Sciences, Bergen, Norway

*Corresponding author: KatharinaStefanieM@gmx.de \& Heli.Routti@npolar.no

This Supporting Information contains the following:

Total number of tables: 2

Total number of figures: 14

Total number of pages: 18 


\section{Supporting Information}

Table S1: The composition of the synthetic mixture used in this study.

\begin{tabular}{c|ccccccc}
\hline \multirow{2}{*}{ Fold } & \multicolumn{7}{|c}{ Concentration $(\boldsymbol{\mu M})$} \\
& pp'DDE & HCB* & tNonachlor & PCB153 & op'DDT & pp'DDD & PCB138 \\
\hline 200 & 44 & $5^{*}(20)$ & 14 & 11 & 10 & 10 & 8 \\
100 & 22 & $2.5^{*}(10)$ & 7 & 6 & 5 & 5 & 4 \\
20 & 4.4 & $0.5^{*}(1.9)$ & 1.4 & 1.1 & 1.0 & 1.0 & 0.8 \\
4 & 0.88 & $0.1^{*}(0.38)$ & 0.28 & 0.23 & 0.20 & 0.19 & 0.15 \\
$1^{* *}$ & 0.22 & $0.025^{*}(0.096)$ & 0.069 & 0.057 & 0.051 & 0.048 & 0.038 \\
0.2 & 0.04 & $0.004^{*}(0.02)$ & 0.014 & 0.011 & 0.010 & 0.010 & 0.008 \\
0.04 & 0.008 & $0.001^{*}(0.004)$ & 0.0028 & 0.0023 & 0.0020 & 0.0019 & 0.0015 \\
\hline
\end{tabular}

* Due to insufficient solubility of HCB, the synthetic mixture was composed of less HCB than average concentrations in fin/blue whales. One-fold of HCB was on average $0.096 \mu \mathrm{M}$ in whale blubber, while in the mixture the concentration was $0.025 \mu \mathrm{M}$. The calculated HCB concentration based on fold of average fin/blue whale blubber contamination data is given in brackets.

** 1 is the median level of contamination data from both, fin whale and blue whale blubber biopsies sampled in Svalbard

\section{Detailed information on the luciferase gene reporter assays}

To harvest COS7 cells, cells were trypsinated and resuspended in Dulbecco`s modified eagle medium (DMEM). The cell density of the suspension was measured using a haemocytometer and adjusted to 50000 cells $/ \mathrm{ml}$. A total of 5000 cells were seeded to each well of a 96-well plate $\left(100 \mu \mathrm{l}, 15600\right.$ cells $\left./ \mathrm{cm}^{2}\right)$ before the cells were incubated at $37^{\circ} \mathrm{C}$ and $5 \% \mathrm{CO}_{2}$. After $24 \mathrm{~h}$ of incubation, COS7 cells were transfected with the reporter plasmid, a control plasmid, and the receptor plasmid by using TransIT-LTI (Mirus BIO LLC, WI, USA). $100 \mu \mathrm{l}$ of the transfection reagent was added to each well of the transfection plate, followed by a $24 \mathrm{~h}$ incubation at $37^{\circ} \mathrm{C}$ and $5 \% \mathrm{CO}_{2}$. Then the growth medium was discarded and $100 \mu \mathrm{l}$ of the DMEM medium without phenol red and $100 \mu \mathrm{l}$ of the medium without phenol red containing 2-fold test concentration of the POP single pollutants or mixtures were added per well. The cells were exposed for $24 \mathrm{~h}$ at $37^{\circ} \mathrm{C}$ and $5 \% \mathrm{CO}_{2}$. After $24 \mathrm{~h}$ of exposure, cells were lysed by the addition of $125 \mu \mathrm{l}$ cell lysis reagent followed by an incubation period of 30 minutes at room temperature with gentle shaking. Cell lysates $(50 \mu \mathrm{l})$ were transferred into two plates. For the ß-galactosidase measurement, transparent 96 well plates were used, for the luciferase activity measurement white 96 well plates were used. For the luciferase measurement $100 \mu \mathrm{l}$ luciferase reagent was added followed by immediate measurement of luminescence (Perkin Elmer, Enspire plate reader). $100 \mu \mathrm{l} ß$-galactosidase reagent was added to the remaining transparent 96 well plate containing lysed cells, incubated for 10 minutes and measured at the absorbance wavelength of $405 \mathrm{~nm}$. 


\section{Supporting Information}

Table S2: Primers used for amplification of THRB, PPARG, GR

\begin{tabular}{|c|c|c|c|}
\hline Gene & Use & Sequence $\left(5^{\prime}-3^{\prime}\right)$ & ID \\
\hline \multirow{4}{*}{ THRB } & \multirow{2}{*}{$\begin{array}{l}\text { Amplification of } \\
\text { initial segment }\end{array}$} & F: CAGTTACTTAGACAAGGACGAGC & MT1252 \\
\hline & & R: CTAATCCTCAAACACTTCCAAG & MT1253 \\
\hline & \multirow{2}{*}{$\begin{array}{l}\text { Amplification of } \\
\text { hinge+LBD w/ } \\
\text { restriction sites }\end{array}$} & F: ATCGAATTCAGCAAGCGTCTGGCCAAG & MT1307 \\
\hline & & R: GATGGATCCCTAATCCTCAAACACTTCCAAGAA & MT1309 \\
\hline \multirow[t]{4}{*}{ PPARG } & \multirow{2}{*}{$\begin{array}{l}\text { Amplification of } \\
\text { initial segment }\end{array}$} & F: GTTGATTTCTCCAGCATTTCC & MT1333 \\
\hline & & R: AATGTTGTCAGTGAACTCGG & MT1334 \\
\hline & \multirow{2}{*}{$\begin{array}{l}\text { Amplification of } \\
\text { hinge+LBD w/ } \\
\text { restriction sites }\end{array}$} & F: ATCGAATTCCATAATGCCATCAGGTTTG & MT1335 \\
\hline & & R: GATGGATCCCTAATACAAGTCCTTGTATATTTCC & MT1336 \\
\hline \multirow[t]{6}{*}{ GR } & \multirow{2}{*}{$\begin{array}{l}\text { Amplification of } \\
\text { part } 1\end{array}$} & F: GATATTCACTGATGGACCCC & MT1326 \\
\hline & & R: CAGCTTCCACAAGTTAAGACC & MT1330 \\
\hline & \multirow{2}{*}{$\begin{array}{l}\text { Amplification of } \\
\text { part } 2\end{array}$} & F: TGAGACCAGATGTAAGCTCTCC & MT1329 \\
\hline & & R: CAGTCACTTTTGATGAAACAGAAG & MT1328 \\
\hline & \multirow{2}{*}{$\begin{array}{l}\text { Amplification of } \\
\text { hinge+LBD w/ } \\
\text { restriction sites }\end{array}$} & F: ATCGAATTCCTGGAAGCTCGAAAAACAAAG & MT1331 \\
\hline & & R: GATGGATCCCAGTCACTTTTGATGAAACAGAAG & MT1332 \\
\hline
\end{tabular}




\section{Supporting Information}

Balaenoptera.acutorostrata.scammoni
Balaenoptera.musculus
Balaenoptera,physalus
Delphinapterus.leucas
Homo.sapiens
Mus.musculus
Orcinus.orca
Rattus.norvegicus
Ursus.maritimus
Balaenoptera.acutorostrata.scammoni
Balaenoptera.musculus
Balaenoptera,physalus
Delphinapterus.leucas
Homo.sapiens
Mus.musculus
Orcinus.orca
Rattus.norvegicus
Ursus.maritimus
Balaenoptera.acutorostrata.scammoni
Balaenoptera.musculus
Balaenoptera,physalus
Delphinapterus.leucas
Homo.sapiens
Mus.musculus
Orcinus.orca
Rattus.norvegicus
Ursus.maritimus
Balaenoptera.acutorostrata.scammoni
Balaenoptera.musculus
Balaenoptera,physalus
Delphinapterus.leucas
Homo.sapiens
Mus.musculus
Orcinus.orca
Rattus.norvegicus
Ursus.maritimus

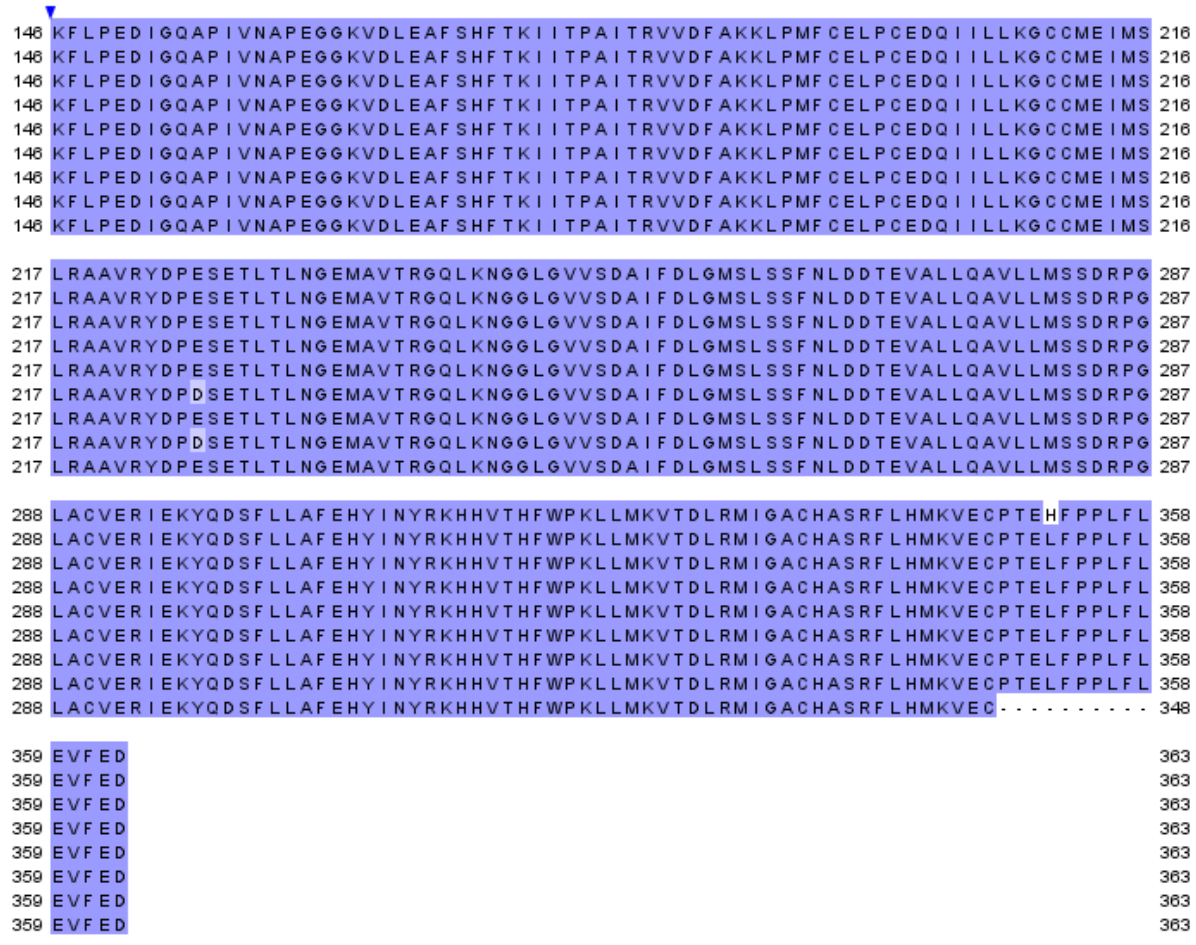

Figure S1: Multiple alignments of THRB LBD sequences. Fin whale (Balaenoptera physalus) and blue whale (Balaenoptera musculus) THRB LBD amino acid sequences were aligned with minke whale (Balaenoptera acutorostrata scammoni), white whale (Delphinapterus leucas), human (Homo sapiens), mouse (Mus musculus), killer whale (Orcinus orca), rat (Rattus norvegicus) and polar bear (Ursus maritimus). The accession numbers are: minke whale THRB isoform X9 (XP_007173928.1, predicted), white whale THRB isoform X3 (XP_022410384.1), human THRB isoform b (NP_001341644.1), mouse THRB isoform X3(XP_011243047.1, predicted), killer whale THRB isoform X1 (XP_012389258.1, predicted), rat THRB isoform 2 (NP_001257783.1) and polar bear THRB (XP_008709785.1, predicted). 


\section{Supporting Information}

Balaenoptera.acutorostrata.scammoni
Balaenoptera, physalus
Delphinapterus.leucas
Homo.sapiens
Mus.musculus
Orinus.orca
Rattus.norvegicus
Urus.maritimus
Balaenoptera.acutorostrata.scammoni
Balaenoptera, physalus
Delphinapterus.leucas
Homo.sapiens
Mus.musculus
Orinus.orca
Rattus.norvegicus
Ursus.maritimus
Balaenoptera.acutorostrata.scammoni
Balaenoptera,physalus
Delphinapterus.leucas
Homo.sapiens
Mus.musculus
Orinus.oraa
Rattus.norvegicus
Ursus.maritimus
Balaenoptera.acutorostrata.scammoni
Balaenoptera,physalus
Delphinaptenus.leucas
Homo.sapiens
Mus.musculus
Orcinus.ora
Rattus.norvegicus
Ursus.maritimus

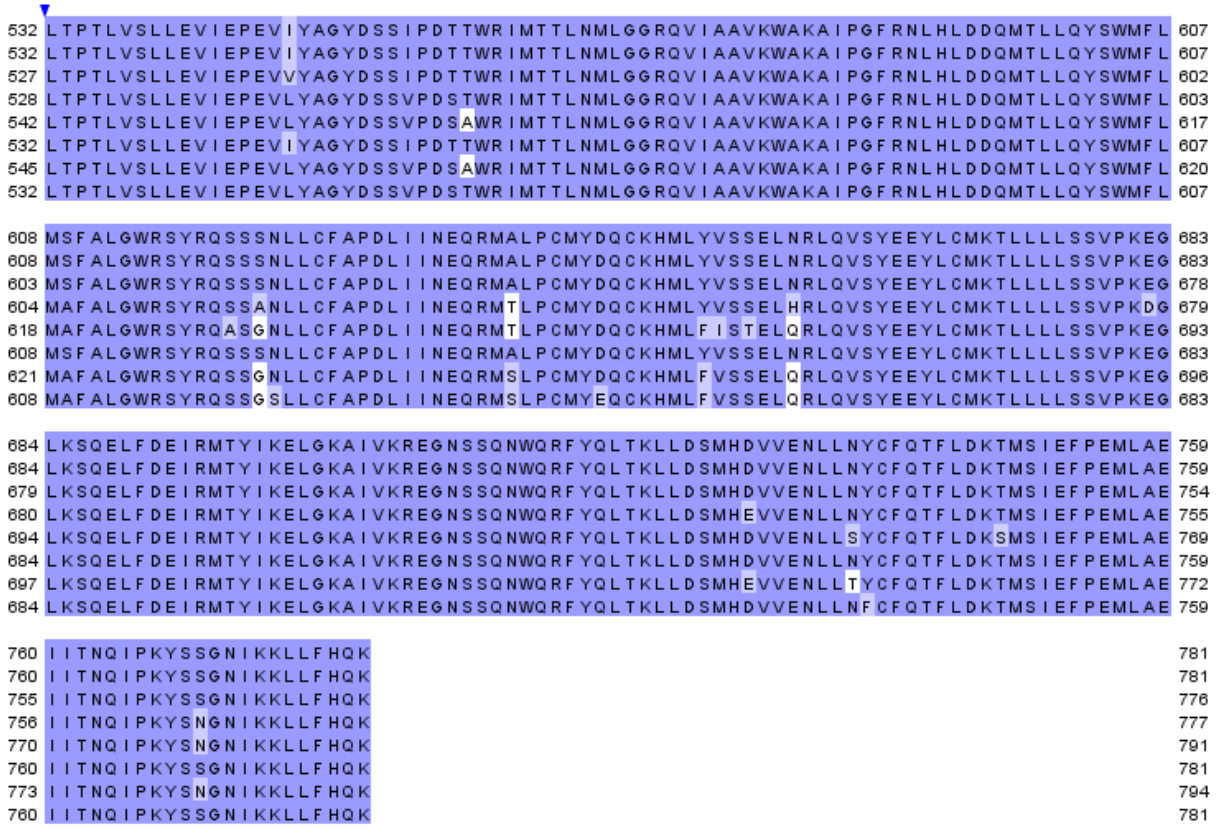

Figure S2: Multiple alignments of GR LBD sequences. Fin whale (Balaenoptera physalus) GR LBD amino acid sequences was aligned with minke whale (Balaenoptera acutorostrata scammoni), white whale (Delphinapterus leucas), human (Homo sapiens), mouse (Mus musculus), killer whale (Orcinus orca), rat (Rattus norvegicus) and polar bear (Ursus maritimus). The accession numbers are: minke whale GR isoform X1 (XP_007194225.1, predicted), white whale GR isoform X2 (XP_022445700.1), human GR (BAH02307.1), mouse GR (ABF57998.1), killer whale GR (XP_004280264.1, predicted), rat GR (AAL66772.2) and polar bear GR isoform X2 (XP_008689674.1, predicted). 


\section{Supporting Information}

Balaenoptera.musculus
Balaenoptera,physalus
Delphinapterus.leucas
Homo.sapiens
Mus.musculus
Orcinus.orca
Rattus.norvegicus
Ursus.maritimus
Balaenoptera.musculus
Balaenoptera,physalus
Delphinapterus.leucas
Homo.sapiens
Mus.musculus
Orcinus.orca
Rattus.norvegicus
Ursus.maritimus
Balaenoptera.musculus
Balaenoptera,physalus
Delphinapterus.leucas
Homo.sapiens
Mus.musculus
Orcinus.orca
Rattus.norvegicus
Ursus.maritimus

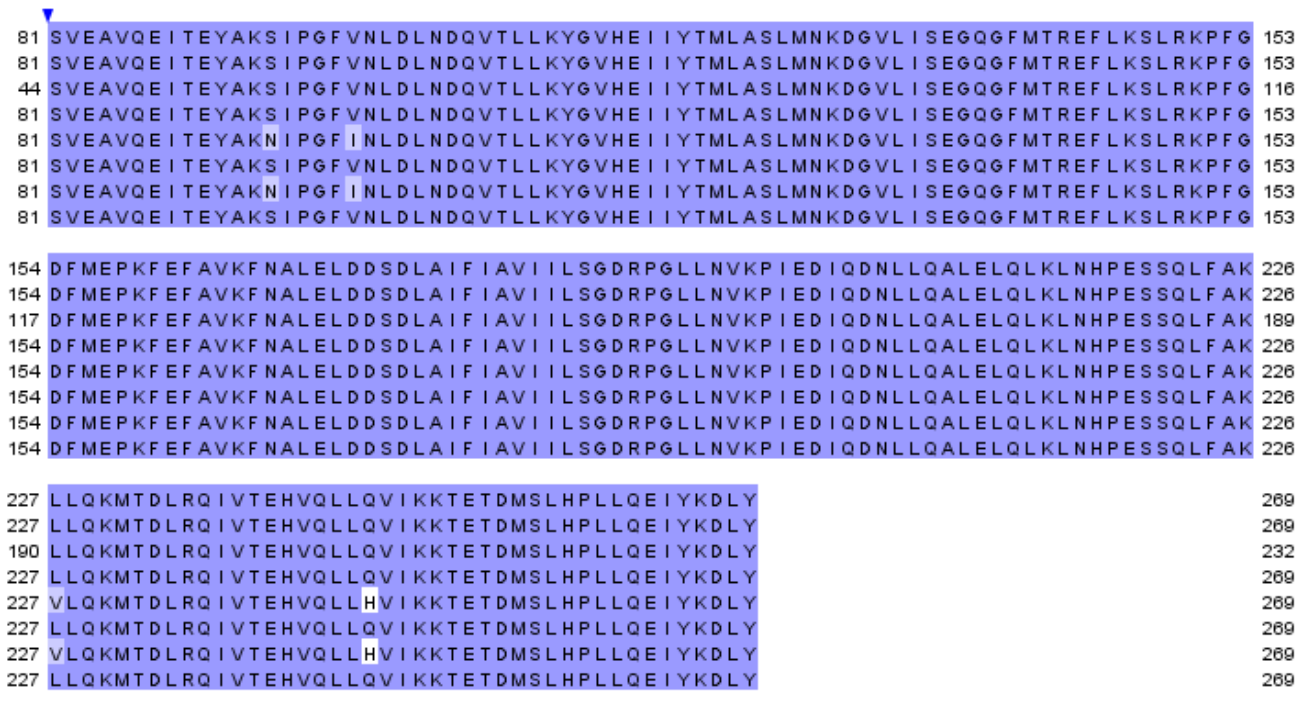

Figure S3: Multiple alignments of PPARG LBD sequences. Fin whale (Balaenoptera physalus) and blue whale (Balaenoptera musculus) PPARG LBD amino acid sequences were aligned with white whale (Delphinapterus leucas), human (Homo sapiens), mouse (Mus musculus), killer whale (Orcinus orca), rat (Rattus norvegicus) and polar bear (Ursus maritimus). The accession numbers are: white whale PPARG isoform 1 (XP_022418477.1), human PPARG isoform 1 (NP_005028.4), mouse PPARG isoform X1 (XP_006505800.1), killer whale PPARG isoform X1 (XP_004284335.1, predicted), rat PPARG isoform 1 (NP_037256.1) and polar bear PPARG isoform X2 isoform 1 (XP_008696091.1, predicted). 

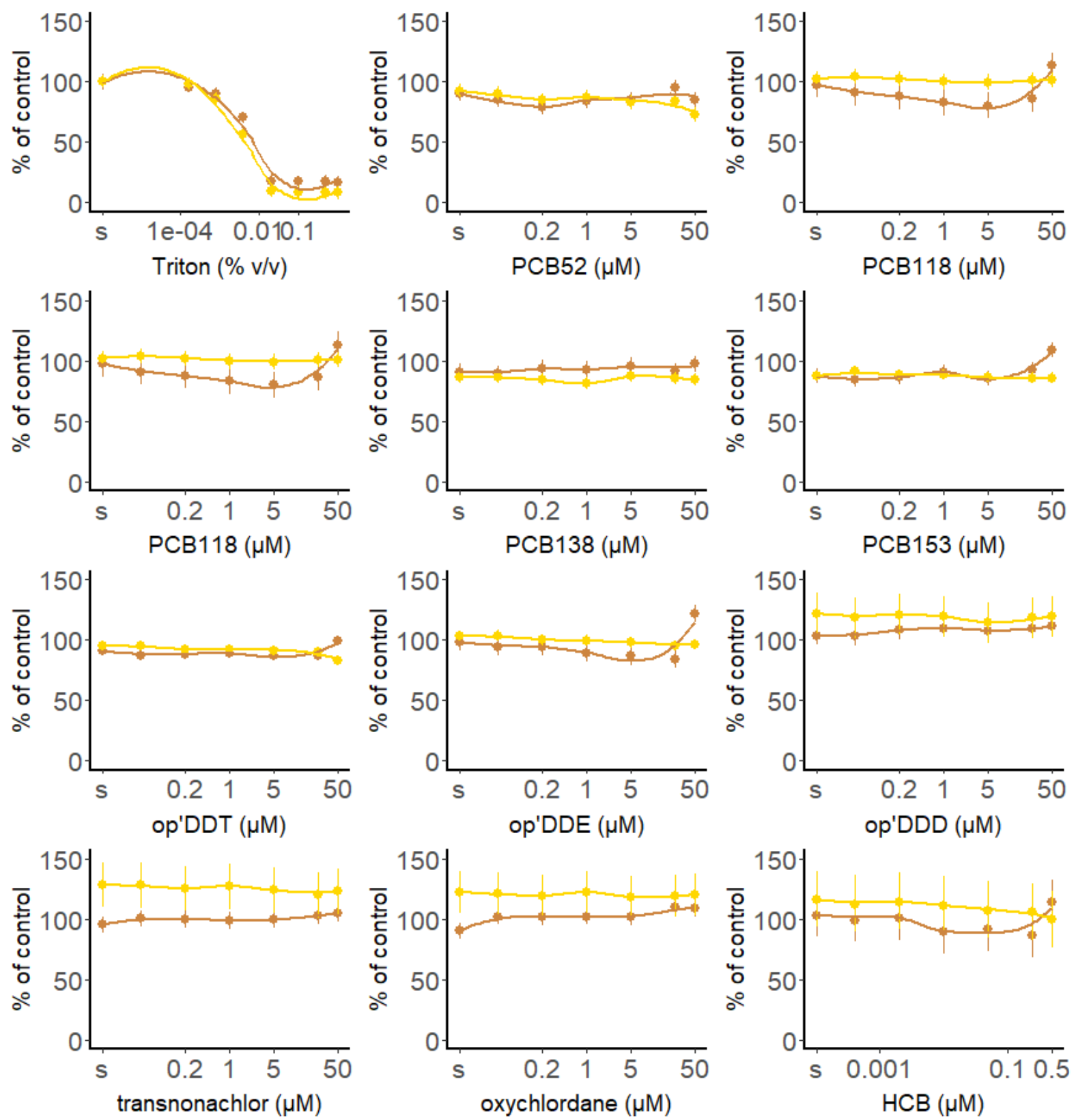


\section{Supporting Information}
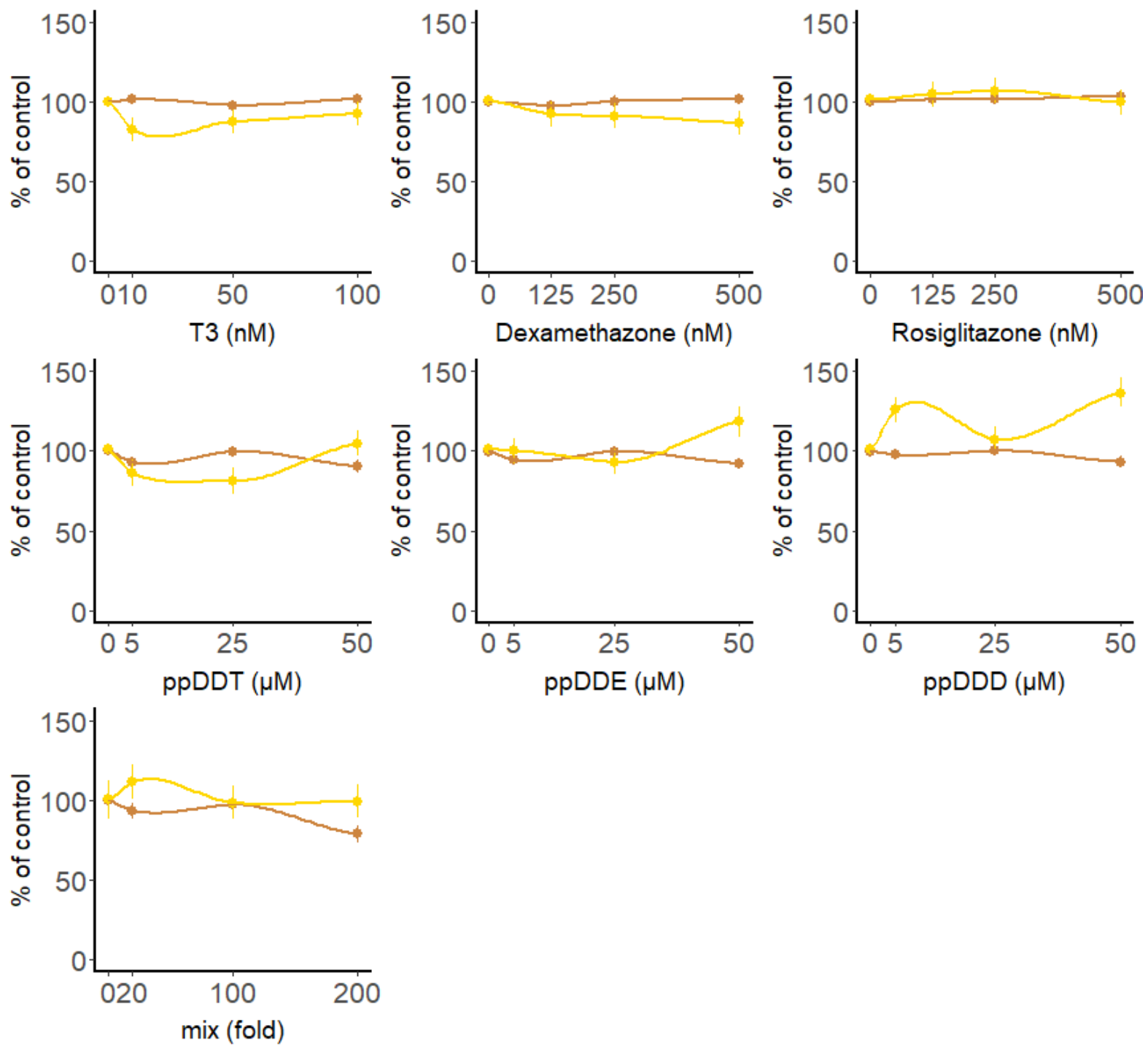

Cytotoxicity $\underset{\text { (membrane) }}{\text { CFDA-AM }} \downarrow \begin{aligned} & \text { Resazurin } \\ & \text { (metabolism) }\end{aligned}$

Figure S4.1: Effect of tested compounds on COS7 membrane integrity and metabolic activity in absence of ligand. Results are presented as percent of control, with $100 \%$ defined as the viability in the presence of only the solvent. At least three independent experiments, each with three replicates, were performed per test compound.

The solvent control is presented as concentration $=0$ or " $\mathrm{s}$ " on the $\mathrm{x}$-axis. 

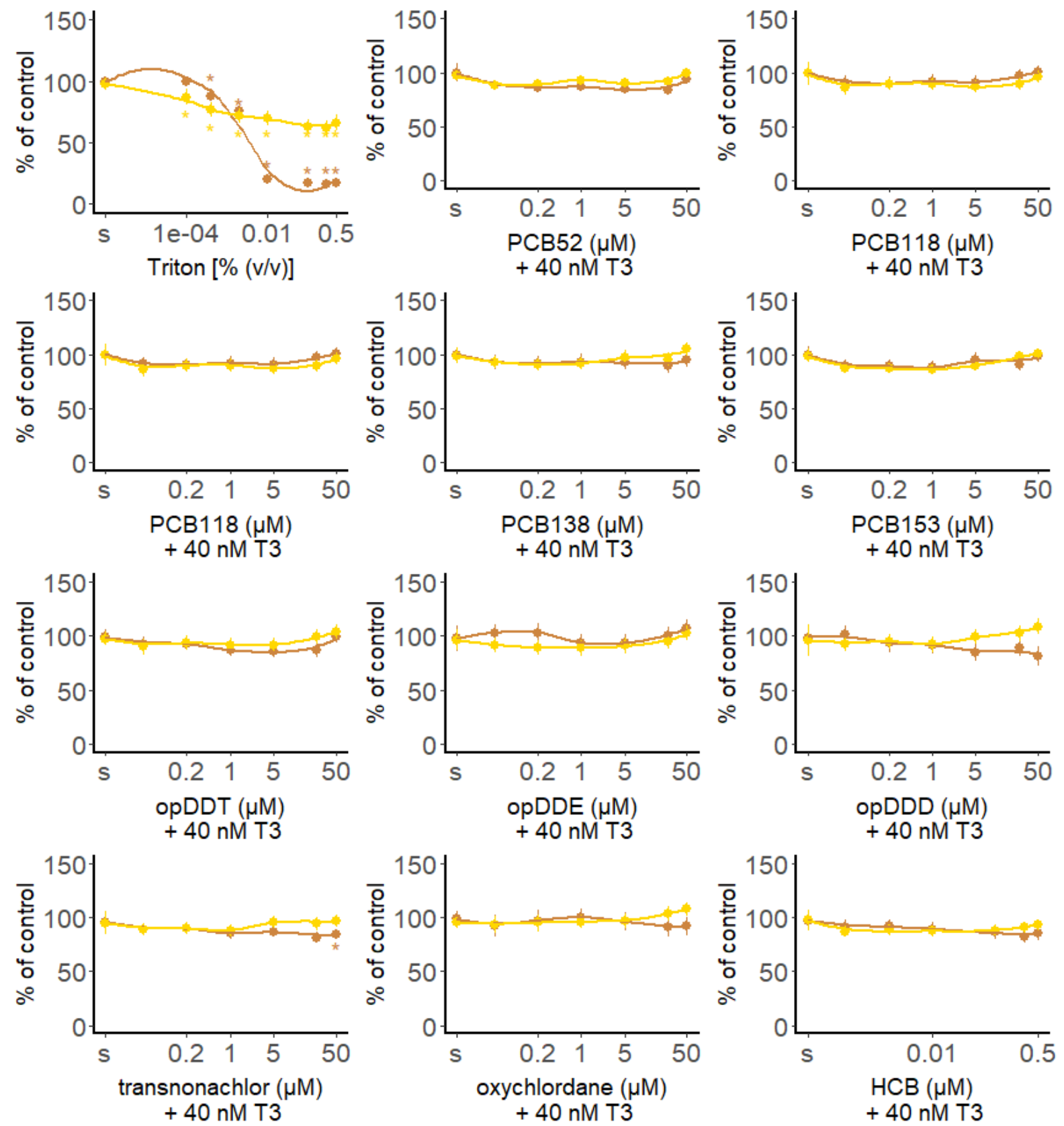

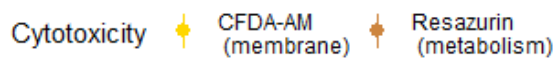

Figure S4.2: Effect of tested compounds on COS7 membrane integrity and metabolic activity in presence of ligand (40 nM T3). At least three independent experiments, each with three replicates, were performed per test compound.

$\mathrm{s}=$ solvent control 


\section{Supporting Information}
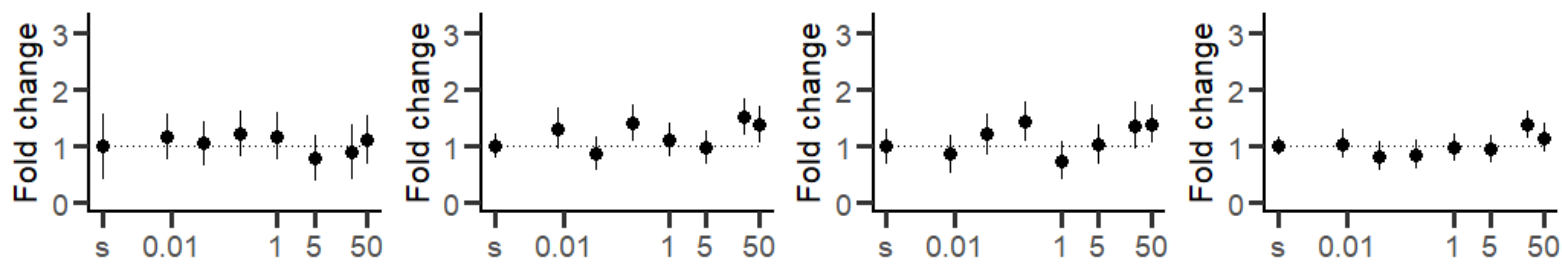

PCB52 $(\mu \mathrm{M})$

PCB101 $(\mu \mathrm{M})$

PCB153 ( $\mu \mathrm{M})$

op'DDT $(\mu \mathrm{M})$
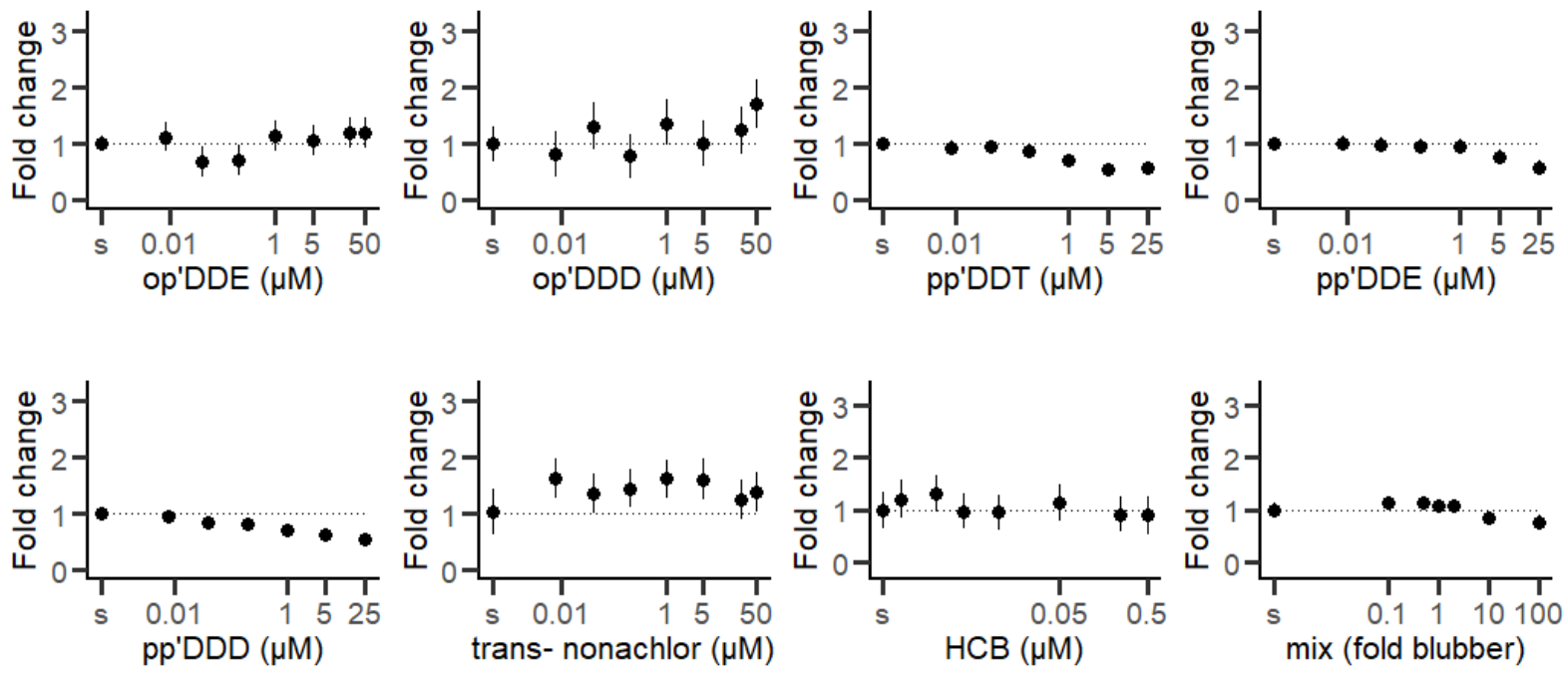

Figures S5: Effect on transcriptional activity of fin and blue whale PPARG. Effects are presented as estimates of fold change over solvent control with 95\% confidence interval derived from linear mixed effect models.

$\mathrm{s}=$ solvent control 

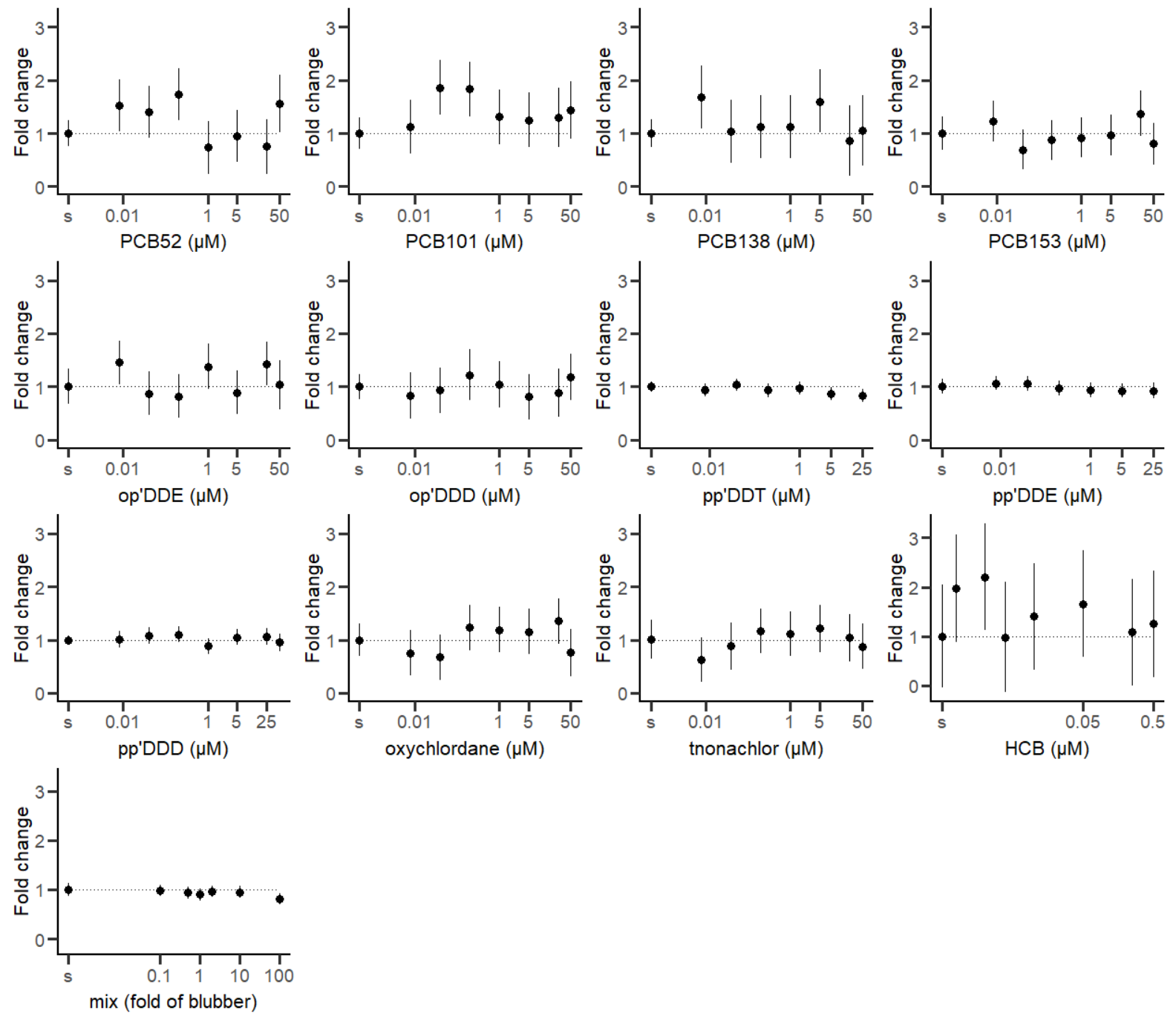

Figures S6: Effect on transcriptional activity of fin and blue whale GR. Effects are presented as estimates of fold change over solvent control with 95\% confidence interval derived from linear mixed effect models.

$\mathrm{s}=$ solvent control 


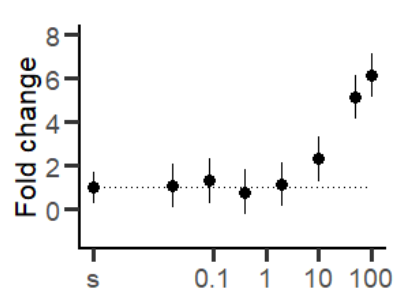

GC-1 (nM)
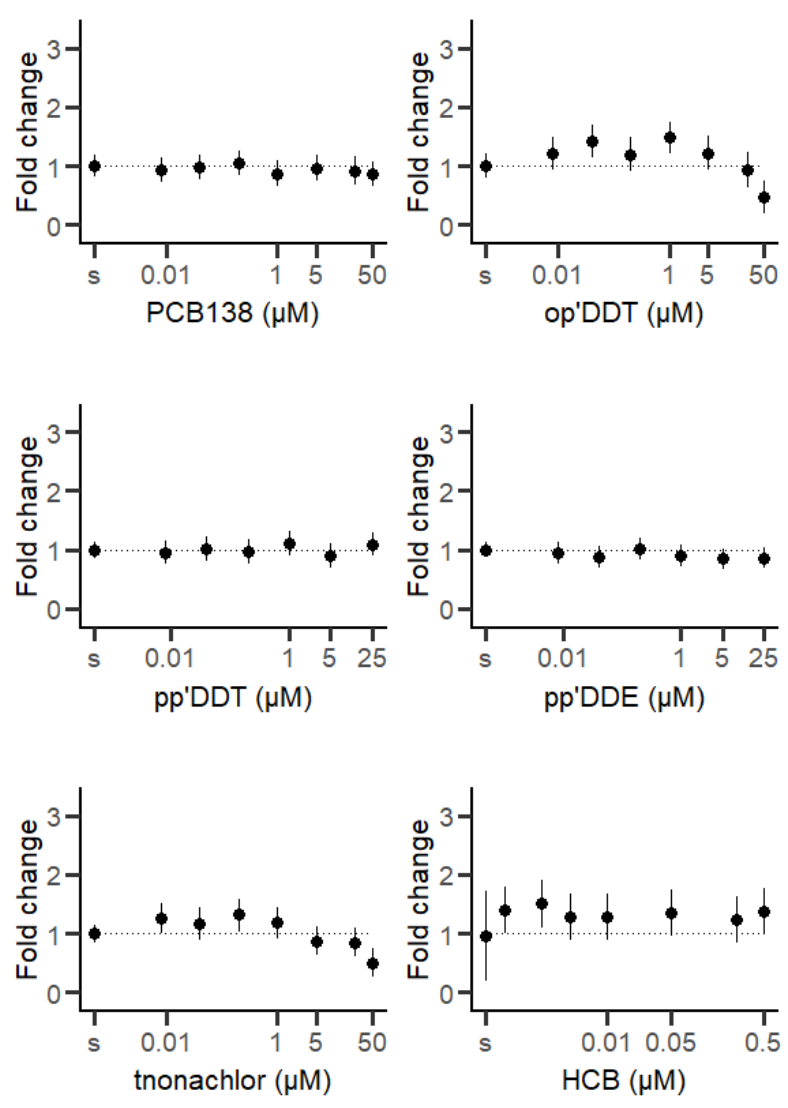

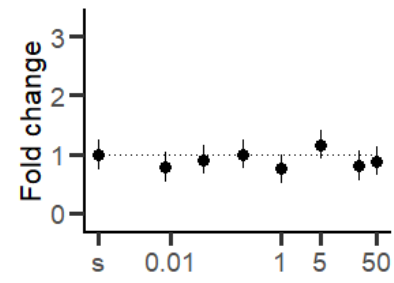

PCB101 $(\mu \mathrm{M})$
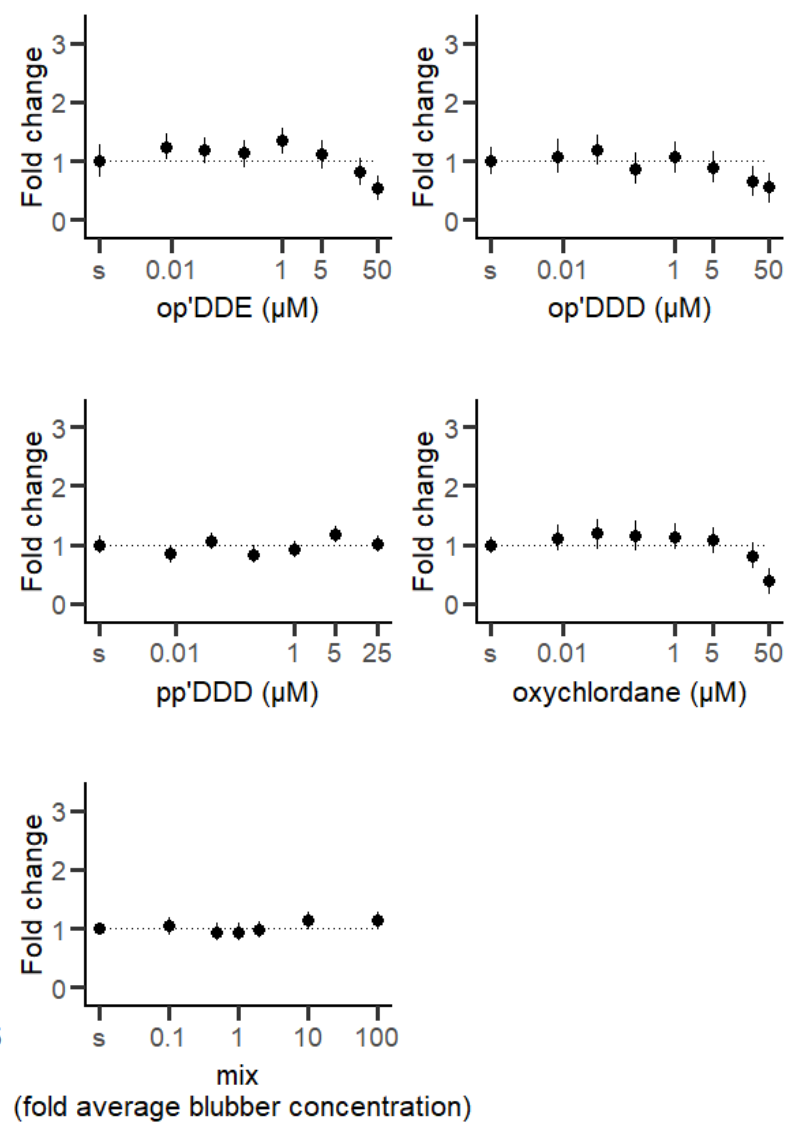

Figures S7: Effect on transcriptional activity of fin and blue whale THRB. Effects are presented as estimates of fold change over solvent control with 95\% confidence interval derived from linear mixed effect models.

$\mathrm{s}=$ solvent control 


\section{Supporting Information}
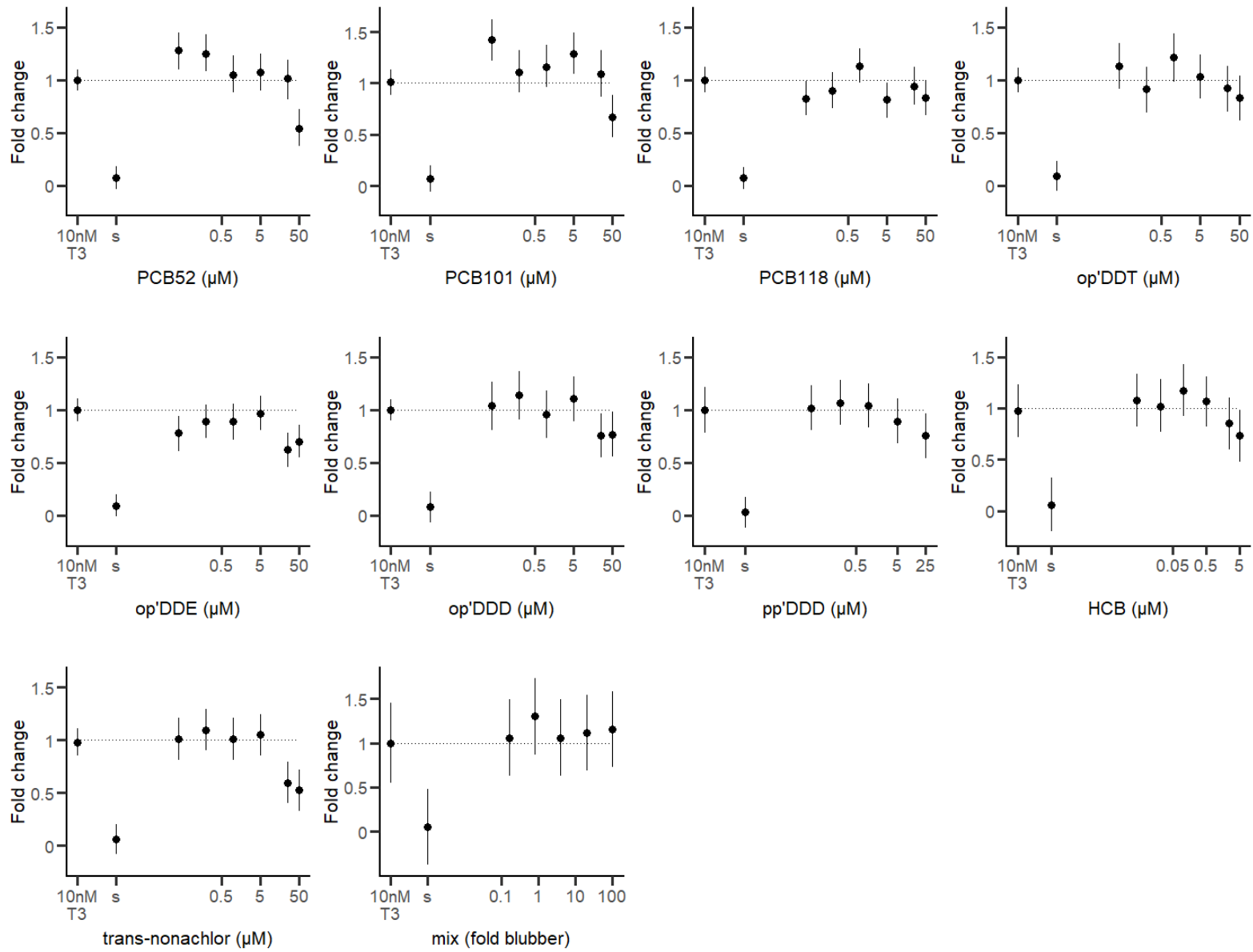

Figure S8: Antagonistic effect by pollutants on fin and blue whale THRB in vitro. Effects are presented as fold change with 1-fold defined as the luciferase activity in presence of $40 \mathrm{nM}$ T3. Data is derived from linear mixed effect models with $95 \%$ confidence interval. $\mathrm{s}=$ solvent control 

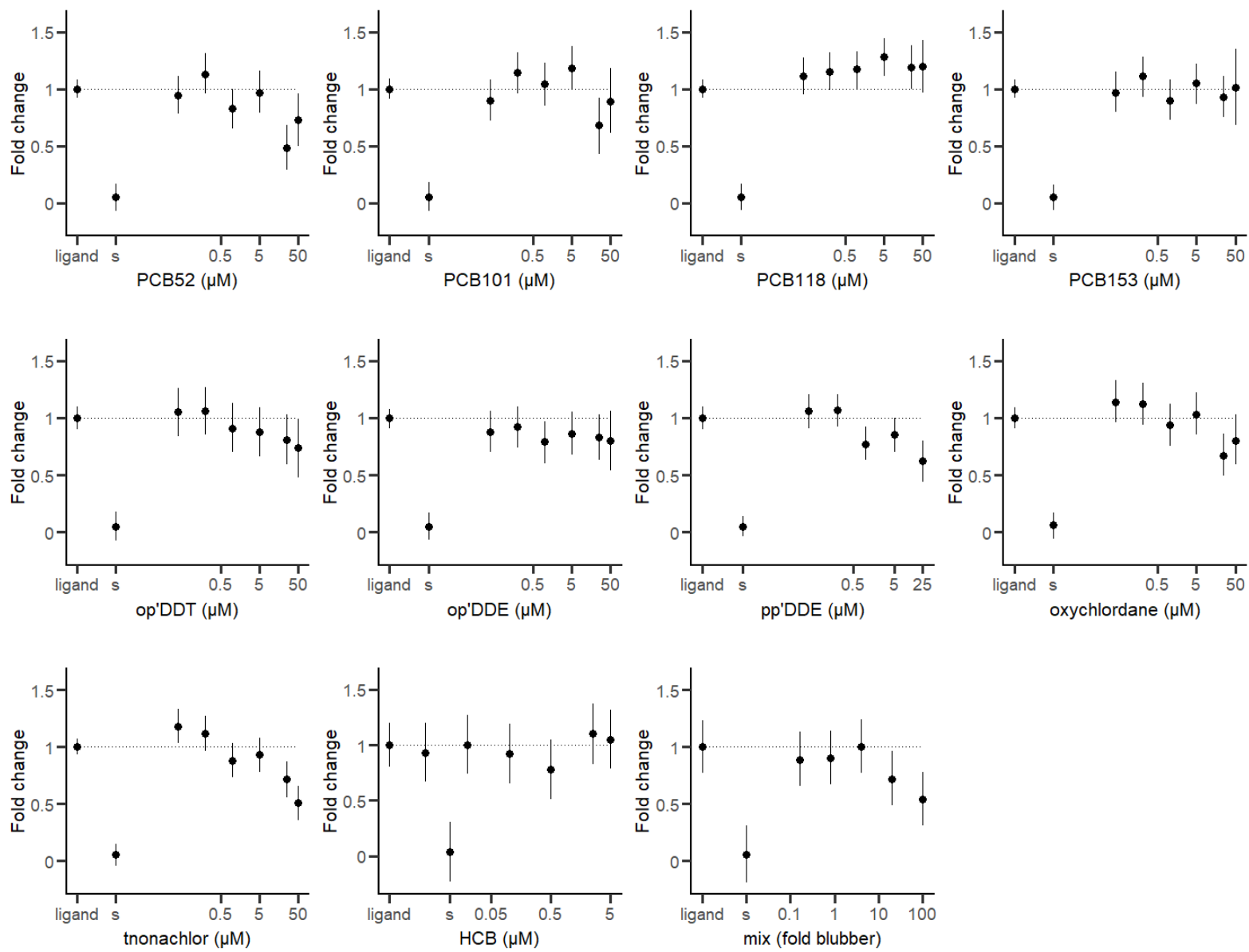

Figure S9: Antagonistic effect by pollutants on fin and blue whale GR in vitro. Effects are presented as fold change with 1-fold defined as the luciferase activity in presence of $10 \mathrm{nM}$ dexamethasone. Data is derived from linear mixed effect models with $95 \%$ confidence interval. $\mathrm{s}=$ solvent control 


\section{Supporting Information}
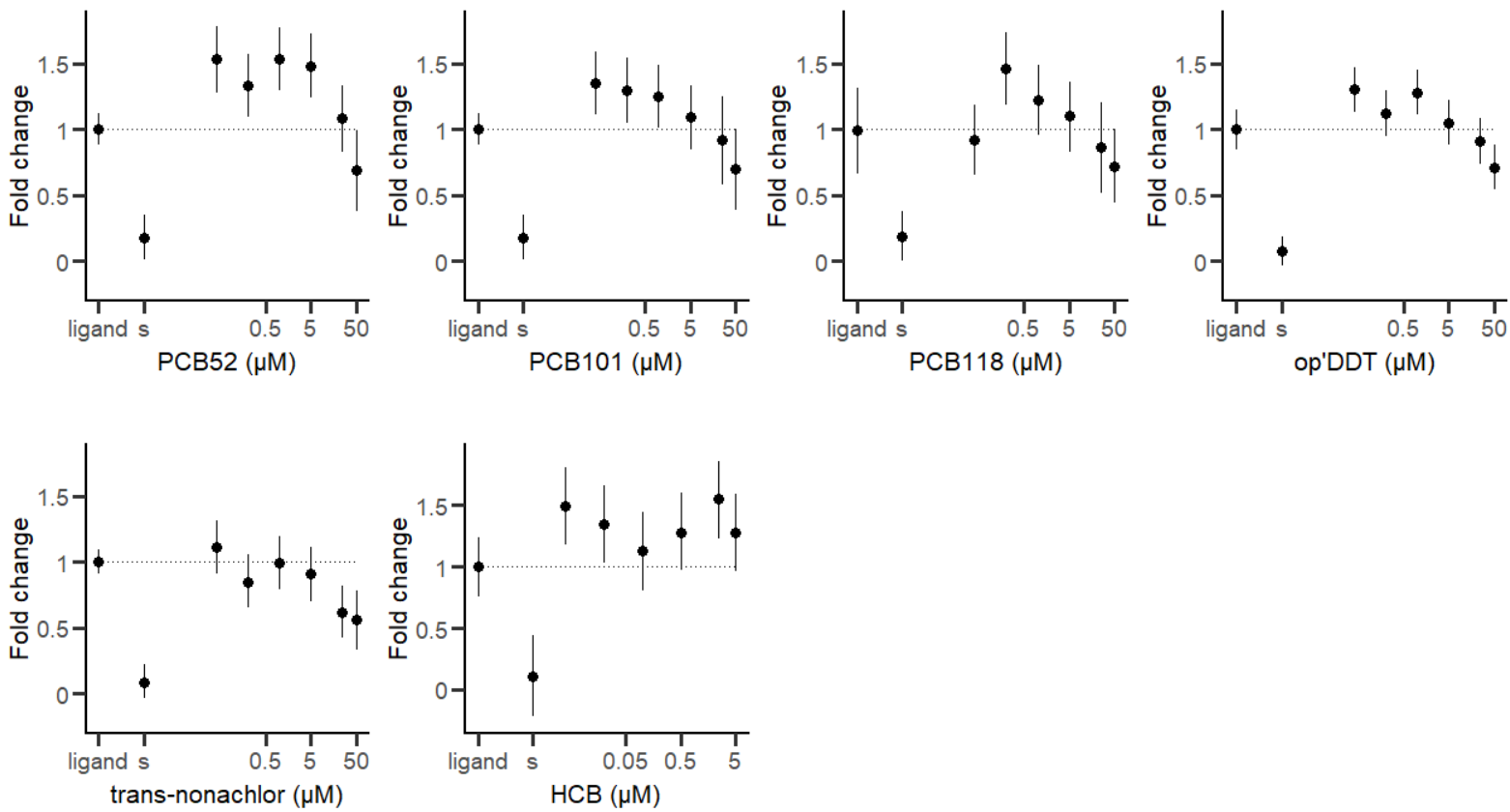

Figure S10: Antagonistic effect by pollutants on fin and blue whale PPARG in vitro. Effects are presented as fold change with 1-fold defined as the luciferase activity in presence of $500 \mathrm{nM}$ rosiglitazone. Data is derived from linear mixed effect models with $95 \%$ confidence interval. $\mathrm{s}=$ solvent control 


\section{Supporting Information}
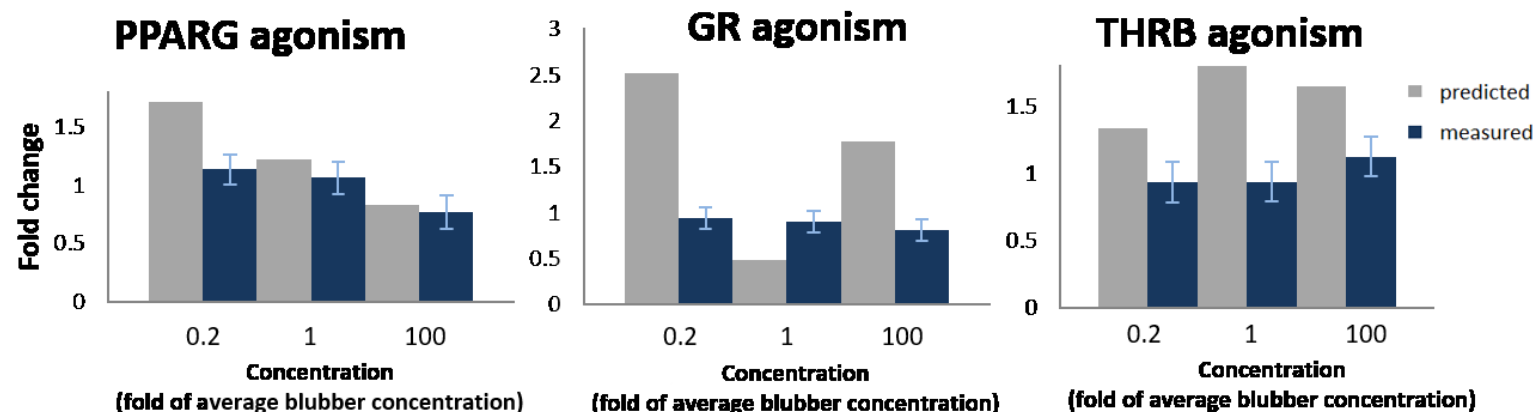

Figure S11: Agonistic effect of the synthetic mixture on transcriptional activity of fin and blue whale nuclear receptors. Measured effects are presented as estimates of fold change over solvent control with 95\% confidence interval derived from linear mixed effect models. Predicted data is the sum of the effects of the single components of the mixture, based on calculations following the concentration summation concept with the assumption of independent action.
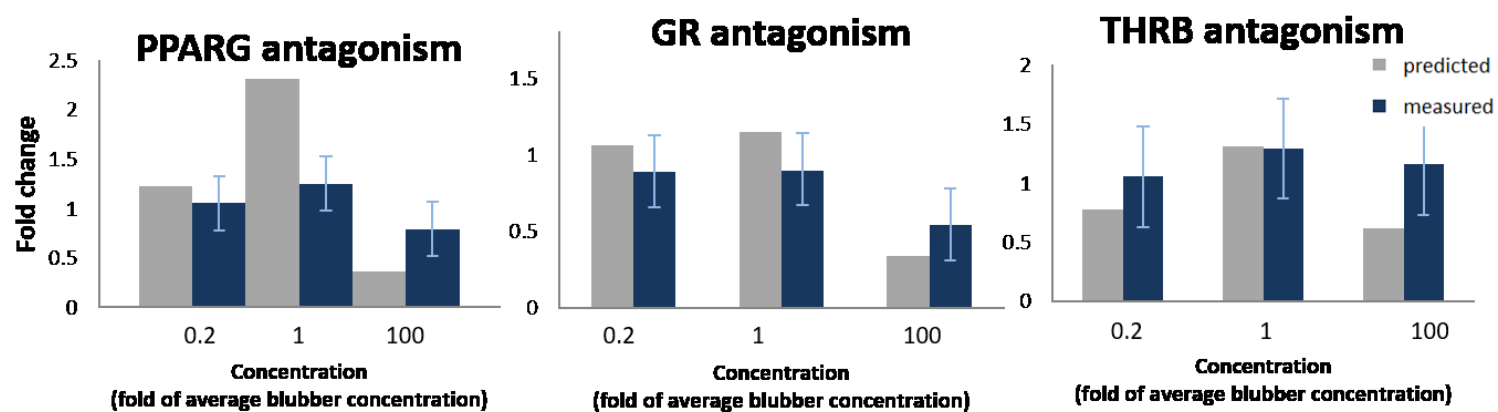

Figure S12: Antagonistic effect of the synthetic mixture on transcriptional activity of fin and blue whale nuclear receptors. Effects are presented as fold change with 1 -fold defined as the luciferase activity in presence of the receptor specific ligand. Measured effects are presented as estimates of fold change over solvent control with $95 \%$ confidence interval derived from linear mixed effect models. Predicted data is the sum of the effects of the single components of the mixture, based on calculations following the concentration summation concept with the assumption of independent action. 

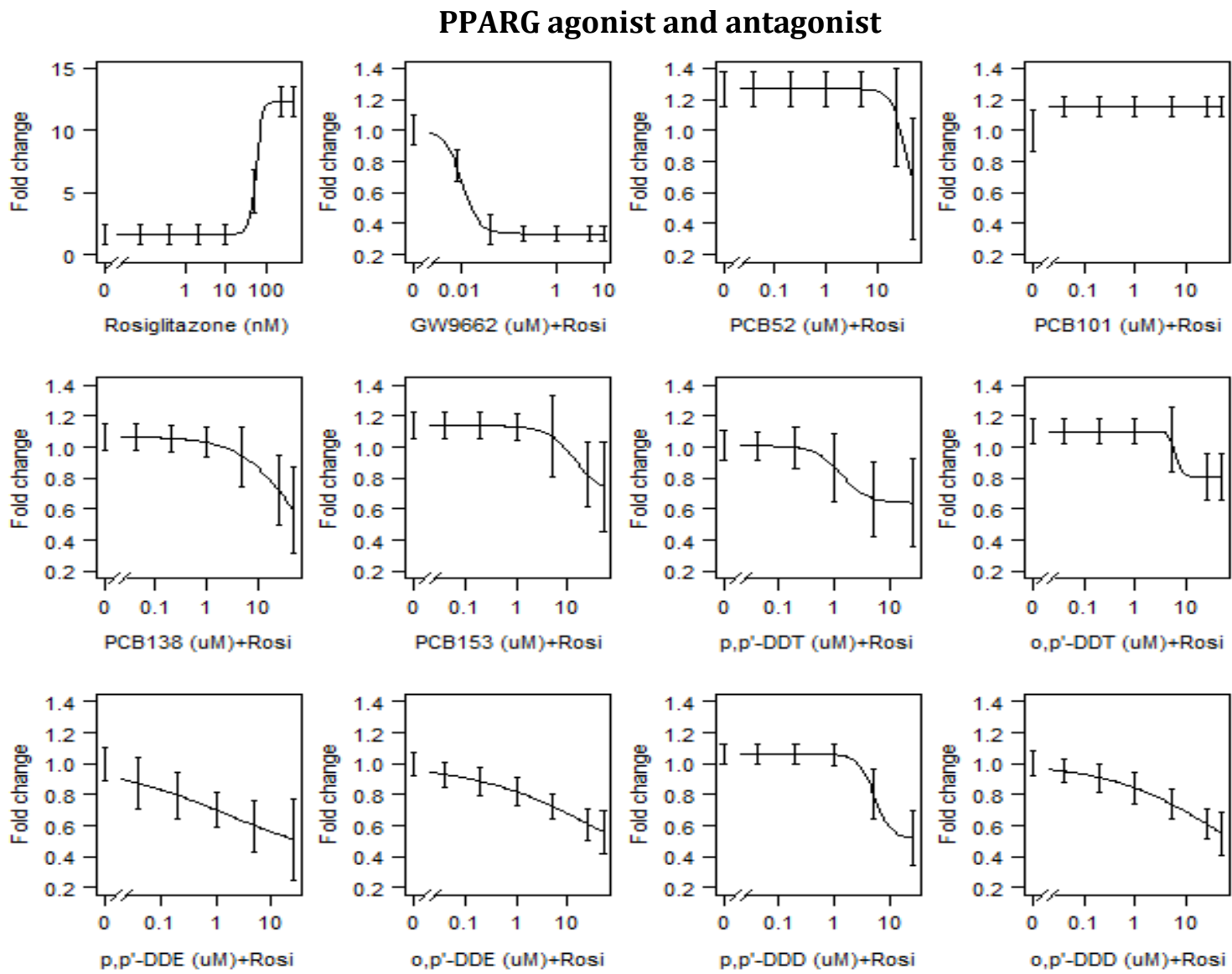

\begin{tabular}{llccc}
\hline Compound & Response & ${\mathrm{EC} / \mathrm{IC}_{50}}$ & Std. Error & $\mathrm{p}$-value \\
\hline Rosiglitazone & Agonist & 57.27 & 18.73 & 0.0028 \\
GW9662+Rosiglitazone & Antagonist & 0.01 & 0.00 & 0.0000 \\
PCB52+Rosiglitazone & Antagonist & 36.78 & 59.47 & 0.5383 \\
PCB101+Rosiglitazone & Antagonist & 0.12 & $\mathrm{NA}$ & $\mathrm{NA}$ \\
PCB138+Rosiglitazone & Antagonist & 47.16 & 262.99 & 0.8581 \\
PCB153+Rosiglitazone & Antagonist & 14.59 & 21.25 & 0.4944 \\
p,p'-DDT+Rosiglitazone & Antagonist & 1.31 & 1.30 & 0.3157 \\
o,p'-DDT+Rosiglitazone & Antagonist & 6.25 & $\mathrm{NA}$ & $\mathrm{NA}$ \\
p,p'-DDE+Rosiglitazone & Antagonist & 0.81 & 1.46 & 0.5806 \\
o,p'-DDE+Rosiglitazone & Antagonist & 45.55 & 165.64 & 0.7840 \\
p,p'-DDD+Rosiglitazone & Antagonist & 5.21 & 1.30 & 0.0001 \\
o,p'-DDD+Rosiglitazone & Antagonist & 40.32 & 132.07 & 0.7609 \\
\hline
\end{tabular}

Figure S13: Dose response curves and $\mathrm{EC} / \mathrm{IC}_{50}$ for compounds that modulated wPPARG transactivation and had sufficient data coverage. Standard errors in the plots and EC/IC50 are derived from four parameter log-logistic models. 

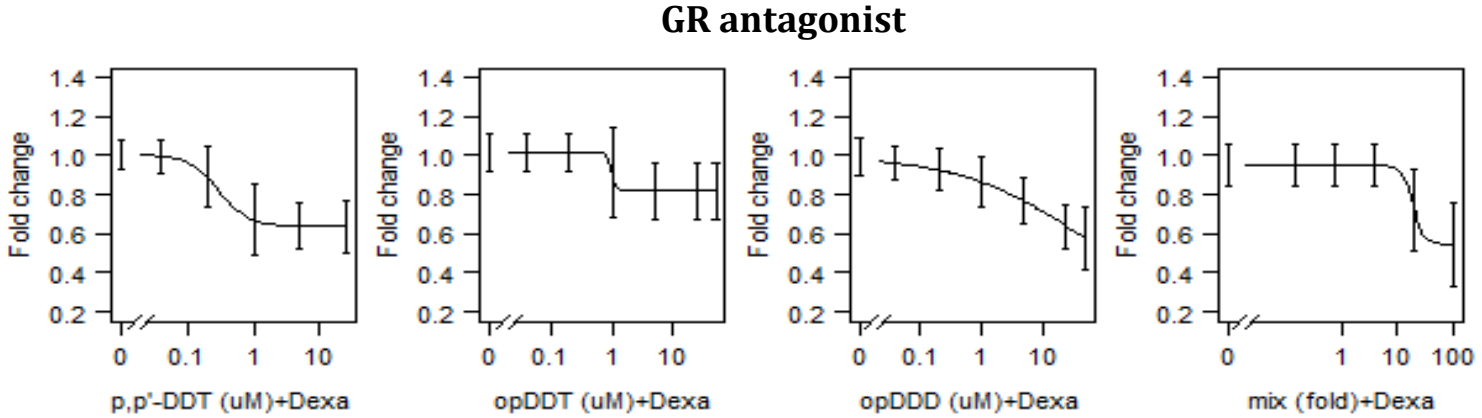

THRB agonist and antagonist
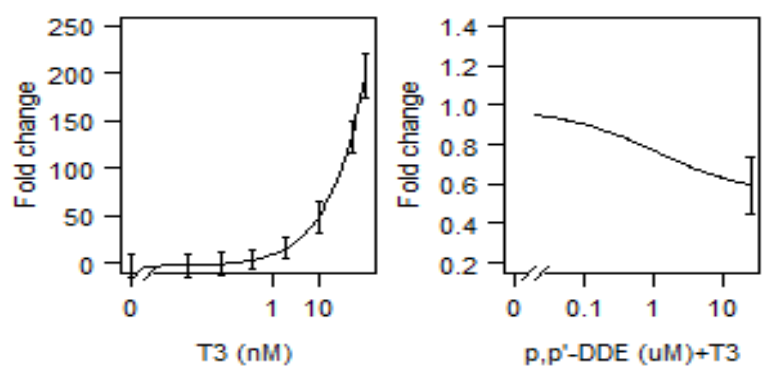

\begin{tabular}{lcccc}
\hline Compound & Response & $\mathrm{EC} / \mathrm{IC}_{50}$ & Std. Error & $\mathrm{p}$-value \\
\hline GR & & & & \\
p,p'-DDT+Dexamethasone & Antagonist & 0.30 & 0.22 & 0.1760 \\
o,p'-DDT+Dexamethasone & Antagonist & 0.99 & 10.18 & 0.9225 \\
o,p'-DDD+Dexamethasone & Antagonist & 40.63 & 147.56 & 0.7837 \\
mix+Dexamethasone & Antagonist & 18.83 & 6.35 & 0.0046 \\
THRB & & & & \\
T3 & Agonist & 835.35 & 1023.23 & 0.4147 \\
p,p'-DDE+T3 & Agonist & 1.12 & 2.09 & 0.5931 \\
\hline
\end{tabular}

Figure S14: Dose response curves and $\mathrm{EC} / \mathrm{IC}_{50}$ for compounds that modulated wGR and wTHRB transactivation and had sufficient data coverage. Standard errors in the plots and EC/IC50 are derived from four parameter log-logistic models. 